\title{
Informacionismo en la integración vertical de archivos
}

\author{
Emilia CURRÁs \\ Universidad Autónoma de Madrid \\ emilia.curras@uam.es \\ http://www.uam.es/emilia.curras
}

Recibido: 08/05/2011

Aceptado: 08/06/2011

\section{RESUMEN}

Se parte de un estudio simplificado de lo que puede ser un archivo, y se articula una definición útil para este caso concreto. Se realza el tipo de documentos allí conservados.

Los archivos proporcionan información. Se articula la diferencia entre dato, documento e información.

Se hace un somero repaso de lo que puede ser información, teniendo en cuenta los quanta de información útil que impactan las neuronas del cerebro, pudiéndose llegar al conocimiento, a la verdad, la certeza y la sabiduría.

Se resalta la gran importancia de la información en el desarrollo del individuo y... de la Humanidad, en su conjunto... De ahí se deduce que es posible formular una nueva teoría epistemológica denominada informacionismo.

Se trata de la Ciencia Unidad y de las relaciones de las distintas ramas del saber, en ella contenida.

Se postula una integración vertical de las ciencias en sí, para pasar luego a ser aplicada a la integración de archivos. Se toma como ejemplo el caso de España. Sólo se hace referencia a cinco de los más importantes archivos. Un esquema intenta dar forma a lo que puede ser una estructura susceptible de esbozar una integración vertical entre ellos.

Los archivos como suministradores de información se pueden encuadrar dentro del Informacionismo. Se postula que la integración vertical de archivos compone una parte del Informacionismo.

Palabras clave: informacionismo, integración vertical, archivo.

Information on the vertical integration of files

\begin{abstract}
It is part of a simplified what can be a file, and articulates a useful definition for this case. It highlights the types of documents preserved there. The files provide information. It articulates the difference between data, documents and information.

It is a brief review of what can be information, taking into account the quantum of useful information that impact the brain's neurons, which may include knowledge, truth, certainty and
\end{abstract}


wisdom. It highlights the importance of information in the development of the individual and ... humanity as a whole... It follows that it is possible to formulate a new theory called epistemological information.

This is the Science Unit and the relationships of the various branches of knowledge contained therein.

It postulates a vertical integration of science itself, then go on to be applied to the integration of. Take the example of Spain. Reference is made only five of the most important files. A scheme intended to give shape to what can be a structure capable of outlining a vertical integration between them.

The files and information providers can be framed within the Information. It is postulated that vertical integration of files composing a part of Information.

Key words: information, vertical integration file.

\section{RAZONAMIENTOS PRIMEROS}

La idea de formular una integración vertical de archivos desde una postulación de una nueva teoría epistemológica, concebida como un Informacionismo, donde el paradigma principal resulta ser la información, quizá, pueda llegar a componer un eslabón superior de abstracción y relación en la concepción y teoría de la ciencia archivística.

Dicho de esta manera se antojan estos enunciados como muy ambiciosos. Téngase en cuenta que esos enunciados generales, conllevan, en sí mismos, una particularización posterior. Un análisis de lo que pueda comportar la ciencia archivística, en su conjunto, conducirá a estudios específicos de determinados casos concretos.

Una vez considerado un archivo en todas sus peculiaridades y aplicaciones, como un almacén, donde se tengan en cuenta tanto sus localizaciones, como sus documentos, funciones y aplicaciones, se podrá llegar a la organización práctica de su integración vertical.

Considerando, igualmente, que, entre otras cuestiones, se puede concebir un archivo como "almacén de datos y documentos" susceptibles de convertirse en información, la relación con el informacionismo nos puede resultar muy comprensible, ilustrativa y útil.

\section{LOS ARCHIVOS}

Los archivos son tan antiguos como la Historia de la Humanidad. Se supone que, allá por las épocas en que se empezaron a anotar, o escribir, datos para su posterior uso, se dispusieron, en algún lugar, para protegerlos y conservarlos. Es de suponer que los archivos diesen origen a la creación de bibliotecas. Quizá los descubrimientos que se vienen haciendo en Ebla (Siria) den evidencia a esta suposición.

La numerosa bibliografía existente muestra muy diversas definiciones, visiones, teorías y aplicaciones prácticas de lo que pueda ser un archivo. No se trata de repetir lo tantas veces dicho por muy numerosos, autorizados, autores; aquí se compone un breve recordatorio que pueda ser útil para razonamientos posteriores. 
El concepto de archivo resulta ser muy amplio y a la vez ambiguo. Hace referencia, por un lado, al edificio donde se localizan una serie de documentos, más o menos ordenados, como, por otro lado, hace referencia a la propia colección de documentos en ese lugar guardados. En los últimos tiempos se encuentran definiciones relacionadas con las técnicas teleinformáticas. También se hace referencia a la acción de archivar y a la profesión de archivero.

En este escrito se considerará el archivo como un conjunto de documentos, continentes de datos, guardados, o almacenados, en un lugar apropiado al caso, conllevando un determinado orden. Ésta es una definición muy simple, pero suficiente para los propósitos de este trabajo. A la hora de desear esquematizar una clasificación de archivos se tendrán en cuenta todas aquellas clases más generales y conocidas, también encontradas en las referencias bibliográficas consultadas.

\section{Propósito de un archivo}

De la bibliografía consultada se puede deducir que los archivos tienen como propósito fundamental guardar y conservar documentos con un fin predeterminado. Pero muy pobre quedaría ahí su función si no se siguiese una utilidad, propia de cada archivo, que se cifraría en proporcionar información a quien de ella esté necesitado.

Bien es verdad que, en tiempos no muy lejanos, un buen número de archivos estaban cerrados al público, aunque éste fuese un estudioso especializado. Hay que comprender que, al igual que en las bibliotecas europeas, los documentos allí guardados solían ser muy valiosos. Por ejemplo, en archivos históricos ciertos documentos no se podían exponer a deterioros y trato descuidado. En un despacho de abogados, por ejemplo, ciertos documentos pueden llegar a ser confidenciales y reservados, que tampoco deberán estar a disposición de cualquier persona ajena al caso.

La idea de archivo cerrado ha empezado a cambiar, cuando han irrumpido en ellos las técnicas informáticas, principalmente, las reproductivas de documentos. Se puede permitir, así, el acceso a ciertos documentos, presentando al posible usuario una fotocopia o una grabación en disco. Esta práctica ya se ha generalizado hoy en día.

En cualquier caso, trátese de un archivo cerrado, o uno abierto, un propósito fundamental deber ser facilitar información a quien necesite de ella.

Se debe hacer hincapié en que en los archivos no se guarda, o conserva, información. Se guardan y conservar documentos de donde sale la información, con una finalidad muy concreta, para uso y usuarios, a su vez, muy concretos.

\section{Dato, documento, información}

Será muy conveniente y adecuado establecer una diferencia entre documento e información, donde no se puede dejar de lado el dato.

En la bibliografía se encuentran, asimismo, una infinidad de definiciones de documento. Por citar un ejemplo, en el libro La Información en sus Nuevos Aspectos, cuya autora resulta ser la autora de este escrito, se citan ya unas cuantas definiciones de varios autores y también de la propia autora. 
Para la finalidad que aquí se intenta conseguir se propone la siguiente definición de documento: representación ordenada y formalizada, de estructura asequible al intelecto humano con el fin de que su uso nos acerque al conocimiento del mundo en que vivimos.

De esta definición puede sugerirse la pregunta referente a desear saber cuáles puedan ser esas estructuras. Aquí se considerará qué son datos; datos contenidos en los documentos. El dato es un quantum de conocimiento coherente y objetivo, carente de valor; es neutro. En otro plano de cosas, L. McCrank piensa que dato es lo que se da en la unidad misma, desde el dígito a la línea, formando un conjunto de líneas y bits. No se cuentan, ni se recolectan. No son actos, ni constituyen evidencia por sí mismos. Son materia prima para constituir una información.

Así pues, cuando el dato sale del documento con una finalidad determinada y una utilidad concreta es cuando se convierte en información.

Sucede, sin embargo, que el uso generalizado de la información ha dado lugar a que, en el lenguaje corriente, se hable de información sin detallar su origen y procedencia, en cuanto a su diferencia con dato y con documento.

\section{Hablemos de la información}

En estos momentos históricos de cambios en las estructuras sociales, está teniendo lugar un proceso de transmutación que afecta a todas las manifestaciones humanas. $\mathrm{Su}$ mayor influencia se deja sentir en los principios conceptuales, relacionados con las bases teóricas de una gran parte de las disciplinas científicas. La información, considerada en su conjunto, como un elemento primordial, también se ve afectada por las transmutaciones que tienen lugar actualmente. El concepto de información se modifica y amplía, incluso se duda de su identidad como ente en sí mismo; por ejemplo, cuando Lutz Herrschaft nos dice que la información es un atributo, una cualidad del objeto, un valor añadido. También se nos advierte sobre su incoherencia y su espejismo.

Sin embargo, detrás de la información se esconde todo un fenómeno propio de los seres vivientes para acomodar su discurrir existencial al medio en que se encuentran. También para adaptar el medio a su condición natural viviente. En los seres humanos, ese proceso adquiere connotaciones muy particulares. Se trata de un proceso ontológico, donde se incluye la inteligencia. Por tanto, adquiere una nueva dimensión que va del ente al entorno y del entorno al ente. Es, pues, vehículo inevitable en un proceso integrador conducente a conformar la sociedad.

En estos últimos tiempos, se han incrementado notablemente los estudios sobre la información, su naturaleza, su idiosincrasia, sus valores intrínsecos, sus aplicaciones y sus repercusiones económicas.

Cuando, en las clases, trato de explicar qué se puede entender por información, empiezo diciendo que es "todo" y "nada" al mismo tiempo. Efectivamente, es "todo" por cuanto que por su utilización, mental o físicamente, se puede llegar al conocimiento, y de ahí, por ejemplo, tomando su lado más pragmático, a la investigación, la ciencia, la sabiduría y la verdad -verdad objetiva, relativa y condicionada-. De este razonamiento se concluye una connotación trascendental de la 
información, pues, siguiendo en esa línea, de la verdad se llega a la evidencia y a la certeza. Por último, se alcanzaría la sabiduría. También será "todo" cuando se considere su uso en la economía, o en la industria.

Por otra parte, cuando se piensa que la información no es "nada", se hace referencia a sus propiedades, como intangible, abstracta, inagotable, no contaminante y reciclable. Lucrecio, en época romana, decía que "la información es, no siendo, pero permite ser". La información no se puede tocar, sin embargo, ahí está; está actuando sobre las neuronas que se impactan por impulsos recibidos del exterior, de manera que éstas se activan confiriendo al individuo -ser viviente- mayor capacidad de razonamiento, incrementando su inteligencia. Cada generación será más inteligencia que la anterior, puesto que está recibiendo información continua y abundantemente.

Los impactos venidos del exterior al cerebro configuran pequeños quanta de información, que inmediatamente siguen unos procesos capaces de elaborar conocimiento, y posteriormente, ideas, convirtiéndose en quanta de información útil. $\mathrm{Y}$ éste es un fenómeno que se viene produciendo desde que el hombre es hombre, o quizá antes. Helmut Arntz asegura que el homínido pasó a hombre, precisamente por sucesivas captaciones y asimilaciones de la información recibida en su cerebro desde el exterior, de su medio ambiente. Precisamente, basándose en estos principios se han elaborado una serie de teorías, denominadas por la autora de este escrito como "Teorías Neuronales de la Información".

La información, como producto humano, se encuadra dentro del Noosistema, perteneciente al Omnicosmos, en la dimensión del Mesocosmos.

\section{INFORMACIONISMO: NUEVA TEORÍA DEL CONOCIMIENTO}

Aunque se admite que, en realidad, estamos en la Era de la Comunicación, ya que la información se mueve y llega a todas partes por medio de la comunicación, es evidente que la información nos rodea e inunda. La información es la base para toda actividad humana, es base de todos nuestros razonamientos, es principio de toda actitud social, es base... Es base para formular una teoría del conocimiento, tomando como paradigma fundamental la información, a lo que la autora denomina informacionismo. Para mejor fundamentar estos razonamientos, se consideran las relaciones que se pueden aplicar entre algunas de las conocidas teorías epistemológicas y las de la información.

Se empieza por el causalismo que dice que no hay efecto sin causa. Efecto $=$ Quanta de información útil, impactando en el cerebro. Causa $=$ Conocimiento.

El realismo concreta que los objetos reales son la base de los conocimientos. Elimina toda suposición, y no admite más que hechos concretos, que, en nuestro caso, se pueden cifrar en los datos, o mensajes, contenidos en los documentos que deberán ser verdaderos y ciertos.

La teoría que más se adapta es la del positivismo, la cual se basa en que sólo los hechos captados inmediatamente por los sentidos y sometidos a una verificación cuantitativa pueden producir conocimiento. Admite, también, una actitud social, pues la captación del mundo exterior puede condicionar nuestro comportamiento. 
Asimismo, es aplicable, en este caso, la teoría de Fernando de Elzaburu, quien basa su teoría de la organización del conocimiento en un cambio de paradigma de su nueva "visión de la realidad". Vivimos en un período de transmutación y, por tanto, los antiguos parámetros de referencia no sirven. Se deben aceptar otros de un nivel de mayor abstracción, que tienen su base en la Teoría de Sistemas. Por otra parte, Norbert Henrichs postula su teoría, asimismo, en un cambio de paradigma, cuando añade a la información una connotación filo-teológica, basada en la adquisición del "saber" -más complejo que "conocer"-; saber subjetivo y relativo, en cuanto a humano, pero absoluto y objetivo en cuanto a su relación con la ciencia -nota de la autora-

El informacionismo conlleva, por una parte, una postura optimista al pensar que se podrá llegar a un mundo más ecuánime y homogeneizado, si se aplican sus paradigmas acertadamente. Al mismo tiempo, conlleva una postura de espera y esperanza, por cuanto que supone una actitud de incertidumbre, en tanto no se conozca la verdad. Asimismo, presupone un principio funcional de actividad pensante, filosófica y científica, con su repercusión en el desarrollo de la ciencia. De igual manera, repercute en actividades cotidianas, como el comercio y la industria. Su influencia se deja sentir en el comportamiento moral y en lo cultural. También influye en actividades pragmáticas, como, por ejemplo, la toma de decisiones. Situarse en esta postura basada en el informacionismo, supone ver el mundo desde un nivel superior, donde se observa una amplitud de conceptos, un grado más elevado de abstracción, donde se relacionan entre sí, aplicando la Teoría de Sistemas.

El informacionismo es objetivo en sí mismo; depende, en cada caso, de razonamientos reales, a su vez objetivos, basados en la existencia de una información, nuevamente, real y objetiva, por ello mismo, verdadera. Por tanto, el informacionismo es, a su vez, verdadero y real. Estas posturas, un tanto deterministas, afirmando una visión totalitarista de la información, como germen de "todo" lo que sucede en el Universo, donde se incluye el planeta Tierra y el resto del Cosmos, conduce a considerar el informacionismo desde un aspecto panteísta, de connotaciones globalizadoras, tenido éste en sentido positivo; así pues, se puede considerar, asimismo, con atributos positivistas.

Se deduce que es posible calificar el informacionismo como humano, objetivo, realista, optimista, globalizante, positivista, filosófico, científico, pragmático, real, terrestre, cósmico..., panteísta. En fin, todos los calificativos "buenos". Existirán investigadores y estudiosos que no estarán de acuerdo con esta clasificación y teorías..., esperemos sus reacciones.

En cualquier caso, se afirma que la época del Informacionismo ha llegado.

\section{CIENCIA-UNIDAD}

Para llegar al concepto de una integración vertical de archivos, y poder plantear una estructura, sea ésta considerada desde cualquier aspecto que se contemplen los archivos, es necesario hacer referencia a la unidad de la ciencia y la propia integración vertical de la ciencia. 
Uno de los puntales sobre los que se apoya la evolución de la Humanidad es la ciencia. La ciencia concebida como unidad de saberes, pero también como camino para llegar a ellos. Precisamente estas ideas suponen ser uno de los primeros indicios del cambio de mentalidad por el que venimos abogando.

Considerar la ciencia como una unidad de saberes no es cosa de ahora. Ya en los postulados del Tao Te Ching se habla de ello. Pero sabemos que las ideas -nunca nuevas y originales- se vienen repitiendo en períodos más o menos largos, recorriendo una curva que presenta máximos y mínimos sobre la línea central, en la que se puede colocar la evolución de la Humanidad. Así desde 2500 años a.C. hasta nuestros días, encontramos, a intervalos variables, las mismas ideas reflejadas en los pensadores que vivieron en las diferentes épocas: Alberto Magno, Hermes Trimegisto, Karl Marx... y más recientemente Schrödiger y Morcillo Crovetto. De entre todos ellos, en nuestros días, se puede citar a Heisenberg, que cifra el desarrollo en la búsqueda de la unidad de la ciencia... V. Weizsäcker nos dice que nuestro espíritu busca la unidad de la ciencia. Alexander King generaliza aún más, cuando combina el conocimiento con la relación próxima entre fenómenos, sucesos y estados de entendimiento.

\begin{tabular}{|c|}
\hline ALGUNOS PENSADORES \\
QUE HAN POSTULADO \\
LA IDEA DE CIENCIA-UNIDAD \\
\hline
\end{tabular}

\begin{tabular}{lr} 
Lao-Tse & s. VI-IV a.C.? \\
\hline Platón & 427-347 a.C \\
\hline Alberto Magno & s. XIII \\
\hline Pedro Calderón de la Barca & 1677 \\
\hline Bodganov & 1923 \\
\hline Henri Poincaré & 1964 \\
\hline Ludwig Von Bertalanffi & 1968 \\
\hline Alexander King & 1976 \\
\hline Aurelio Peccei & 1984 \\
\hline Ilya Prigogine & 1988 \\
\hline Fernando de Elzaburu & 1988 \\
\hline Mario Bunge & 1988 \\
\hline Emilia Currás & 1988 \\
\hline J.J. Scala Estaella & 1991
\end{tabular}

Figura 1 


\section{INTEGRACIÓN VERTICAL DE LAS CIENCIAS}

La ciencia es una y plural, a la vez, por cuanto se compone de las distintas ciencias en un proceso de integración e interrelaciones de alta complejidad. Complejidad que va aumentando conforme avanza el proceso de evolución, tanto de la Humanidad, como de todo el fenómeno vital sobre nuestro planeta, y el devenir del mismo cosmos. Hasta ahora se ha venido estudiando este proceso de integración desde una perspectiva horizontal. Admitiendo que todas las manifestaciones del intelecto humano y todos sus logros tienen cabida bajo un aspecto determinado de la ciencia, es decir, de alguna de las ciencias que la componen, por ejemplo, las ciencias históricas, jurídicas, químicas, biológicas..., se entiende una concordancia entre ellas relacionándolas en planos situados a un mismo nivel. Esta postura implica grandes dificultades cuando lo que realmente se pretende es buscar soluciones a la situación de crisis que venimos viviendo, y preferentemente en la organización del conocimiento.

Ahora bien, cuando lo que se desea es estudiar las interrelaciones de unas ciencias con otras, en unos procesos de integración que proporcionen visiones globales de donde extraer soluciones prácticas y locales que remedien nuestros males en cualquier aspecto de nuestra vida, ya sea en ciencia de la información, social, económico..., entonces tenemos que variar nuestros esquemas, tenemos que cambiar de mentalidad; tenemos que acceder a un nuevo nivel.

Probemos, pues, al menos, considerando la integración de las ciencias en un sentido vertical.

Cada una de las distintas ciencias compone un eslabón dentro del conjunto de la unidad de la ciencia. Ese eslabón viene influenciado por distintos eslabones, ciencias, en mayor o menor grado, según el ámbito de conocimiento de cada una de ellas en relación con las demás, o con aquélla concreta, objeto de un estudio determinado. Para acceder al conocimiento completo de cada una de las ramas de la ciencia, se debe partir de aquélla que pueda servir de base e ir subiendo o bajando, introduciendo otras ciencias que impliquen mayor complejidad, o medios complementarios, dentro del ámbito del saber a que haga referencia nuestra ciencia de estudio.

Se ha de convenir en que las distintas ramas del saber humano que componen la unidad de la ciencia, se influencian unas a otras en distinto grado y a diferentes niveles. Pongamos como ejemplo, la química que viene influida por la física, por la biología, por la mineralogía, por la historia... Por su parte, la agricultura recibe influencias de la química, la meteorología, la zoología...En la psicología hay que tener en cuenta las influencias de la sociología, la moral, la biología; en la información interviene la comunicación, la electrónica, la filosofía... Asimismo, hemos de convenir en que unas ciencias se presentan como base o fundamento para llegar a un pleno conocimiento de una ciencia particular. Así, la física es base para el estudio de la química; la medicina para el estudio de la psicología; la botánica es el fundamento de la agricultura. También se debe admitir que ciertas ciencias intervienen en el desarrollo del resto de las ramas del saber. Parece obvio que la historia, las matemáticas -sobre todo en su aspecto práctico y estadístico-, la geografía, la ética, la información... son ciencias que deben ser incluidas en el estudio de cualquier ciencia. El grado de dependencia e influencia de 
una ciencia en otras varía, según los casos, de manera que el entramado de relaciones entre ellas se presenta complejo en interrelaciones dialécticas concordantes y discordantes que evolucionan en el espacio y en el tiempo. Su estudio se realiza aplicando la Teoría de Sistemas.

Leyendo obras concernientes a los estudios sobre la ciencia y otras cuestiones diversas, se encuentran referencias a estas ideas sobre la integración vertical de las ciencias, aunque quizá sin tener conciencia de ello. Así, por ejemplo, ya en el Tao se dice que, cuando se considera que las cosas se integran, se puede alcanzar la naturaleza del Cielo, la Tierra y todas las cosas. Aquí la integración se realiza en sentido descendente, desde lo sobrenatural -quizá solamente universal- hacia lo natural conocimiento de la Naturaleza-, hasta lo social, económico, político -que componen "todas las cosas"-. Eric Schwartz habla de la integración hombre-cosmos. Aquí se sobrentiende que la relación de las partes de la ciencia se realiza en una integración vertical, ascendente, justamente de sentido contrario a la teoría anterior. Paolo Manzelli postula que la física clásica no sirve para entender la relación energía-materiainformación. Para ello, es preciso recurrir a introducir procesos intermedios graduales en su complejidad, donde los procesos químicos juegan un papel importante. En este sentido, F. de Elzaburu asegura que de la física deviene la biología, suponiendo un salto vertical de nivel desde aquélla, tomada como base, a ésta tomada como colofón final.

Por su parte, Mario Bunge propone que todos los sistemas poseen cinco aspectos interdependientes: cultural, político, económico, biológico, ambiental..., es decir, cinco manifestaciones de otras tantas ramas de la ciencia en ascendente nivel de complejidad. Bohm y Chew inciden en que la "conciencia" -ciencias morales- deberá ser incluida en la futura teoría de los fenómenos físicos -ciencias físicas-, si se desea dilucidar el verdadero aspecto esencial del Universo. Estos autores coinciden en sus ideologías fundamentales con las teorías de Fritjof Capra, no muy lejos de los principios del Tao de la Física. Desde otro punto de vista, Karl Popper concibe la psicología como disciplina dentro de la física.

Como ejemplos sean éstos suficientes para poner de manifiesto que subyace, en la mente de varios de nuestros pensadores actuales, la idea de la integración vertical de las ciencias.

\section{INTEGRACIÓN VERTICAL DE ARCHIVOS}

Siempre se tiende a seguir las costumbres y prácticas establecidas como válidas. Aparentemente éstas pueden dar buenos resultados en determinados momentos. Cuando se ha intentado integrar archivos, se ha intentado llevarlo a cabo utilizando los métodos habituales, que han venido siendo válidos considerando esa integración en sentido horizontal. Al igual que en la ciencia, tenida como Unidad de Saberes, esa integración horizontal se ha quedado un tanto corta. Se deben considerar muchos más parámetros y condicionantes que en tiempos pasados. La evolución de la Humanidad circula por unos derroteros donde todo se ha vuelto más complejo. Nuevos inventos, nuevos descubrimientos, nuevas prácticas, nuevas teorías..., todo influye para contribuir a 
llegar a la complejidad actual, en cualquier campo del Saber Humano, donde, naturalmente, las tecnologías electrónico-informáticas han jugado un papel importante.

Aquí se intenta ver si sería posible solucionar algunos de los problemas, y peculiaridades que se presentan, tanto en la Ciencia Unidad en sí misma, como en cada uno de sus componentes. Incluso se intenta, asimismo, llegar a proponer soluciones a cuestiones muy concretas. Y esto se trata de conseguir aplicando una integración vertical de aquello, que, en cada caso, se desea integrar.

En un trabajo titulado "Integración Vertical de las Ciencias", publicado en varias revistas españolas e inglesas, se establecen los principios fundamentales de esa integración vertical, aplicada a la Ciencia como Unidad de Saberes; a la Ciencia Unidad. Este artículo es el que ha servido de guía y modelo para aplicar la integración vertical a los archivos.

La ciencia archivística es sumamente compleja, por lo que a la hora de querer establecer una integración vertical de los archivos, habrá que ir dividiendo cada uno de los aspectos, o apartados-clasificaciones, etc., que se puedan considerar en un archivo.

En un archivo, en particular, se deberán tener en cuenta, primeramente, los tipos de documentos; a continuación, igualmente, los sistemas de ordenación y clasificación, y las maneras de acceso, las formas de difusión de la información, el tipo de usuarios...

En el orden funcional, se deberán considerar los aspectos prácticos, donde se habrá de incluir al personal, en todas sus categorías, como asimismo habrá que pensar en el material de archivo y de oficina, material informático, tangible y material fungible, etc., etc.

En este caso concreto, se va a prescindir de los aspectos funcionales, que pueden ser semejantes, en todos los archivos, sean cuales sean sus aspectos temáticos. Se va a atender más a la temática referida a los tipos de documentos que se conservan y archivan en cada archivo, en particular.

Se tomará el ejemplo de España. Se advierte que la relación de archivos y sus tipos no es exhaustiva, pues, por un lado, es muy arriesgada esta cuestión, y, por otro, se conseguiría una integración muy complicada que traería confusión y no aportaría ningún resultado inteligible.

También se debe pensar que una integración vertical de archivos fuese panacea universal para resolver todos los problemas que los archivos de una nación conllevan.

Ruego a los archiveros que perdonen mi osadía y atrevimiento al tratar el tema; solamente se pretende plantear una posible integración de ciertos archivos españoles, que pueda servir de ejemplo para ilustrar lo que se viene aquí razonando.

\section{CONSTRUCCIÓN DE LAS COLUMNAS}

Así pues, se hace una relación de algunos de los archivos más conocidos, que existen en España: Archivo Histórico Nacional, Archivo de Simancas, Archivo de Indias, Archivo de Protocolos, Archivo de la Biblioteca Nacional, Archivo del Ministerio de Educación, Archivo del Museo del Prado.

Para mejor comprender una integración vertical de aquello que se desee integrar se deberán construir unas columnas, donde figuran en su interior, a modo de ladrillos -o 
cuadradillos- cada una de las partes de que se compone aquello que se desea integrar. Se habla de ladrillos por aquello de construir columnas. En la representación gráfica, resulta más inteligible usar la palabra ladrillo, que parece muy intuitiva.

Téngase siempre en cuenta que no se intenta presentar ni una teoría ni método absoluto y exhaustivo. Pero lo que se pretende es hacer comprender estas prácticas lo más sencillamente posible; de ahí que eche mano de muchas simplificaciones.

A la hora de construir las columnas correspondientes a los citados archivos españoles, se sigue la práctica de la simplificación -se ruega perdón, insisto-; incluso sólo se van a considerar cinco archivos: el Archivo Histórico Nacional, el de Simancas, el de Indias, el de Protocolos, y el de la Biblioteca Nacional. Cada uno dedicado a tipos de documentos que difieren entre sí y que, por tanto, conllevan ciertos métodos de clasificación, archivo y catalogación muy sui generis. Y es aquí donde se puede intentar aplicar una integración vertical entre ellos.

\section{ESQUEMA SIMPLIFICADO DE POSIBLE INTEGRACIÓN}

DE ARCHIVOS, SEGÚN SUS PECULIARIDAES

\section{A.H.Nacional Archivo Simancas Archivo Indias Archivo Protocolos Archivo B.N.E.}

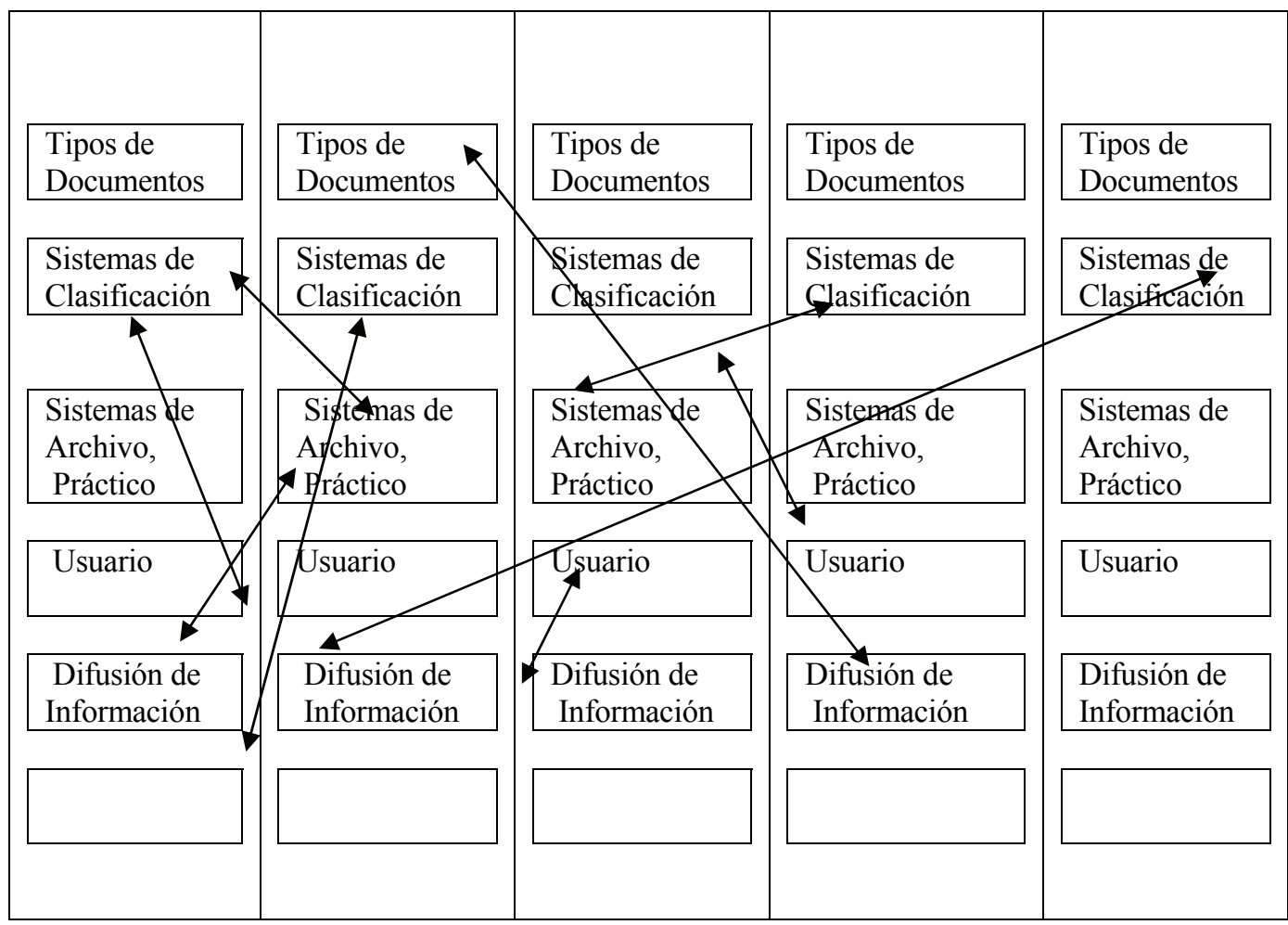

Figura 2 
En la Figura 2 se han representado las diferentes columnas, una por cada archivo, con sus ladrillos correspondientes. Obsérvese que la característica diferenciadora radica en el tipo de documentos que se archivan en cada archivo, en particular. También los tipos de usuarios que acuden a ellos en busca de información, como asimismo los sistemas clasificatorios, archivísticos y de catalogación.

De alguna manera, las distintas formas de trabajo de cada archivo, referidas a los sistemas clasificatorios que utilizan, como también los sistemas propios de archivo, o sus métodos de catalogación, deberían estar, en cierto modo, relacionados entre sí. Igualmente, los distintos tipos de usuarios se podrán relacionar con aquellas cuestiones anteriores. Esto significa que los ladrillos dibujados en el interior de las columnas deberían interaccionar mutua y recíprocamente. Se tendrían que considerar "influencias concordantes y discordantes" a la vez.

No se pretende seguir prácticas reiterativas, pero se opina que se debe volver a insistir en las simplificaciones empleadas en este escrito. Por consiguiente, en el esquema de la Fig. 2 no se dibujan todas las interacciones-líneas que se deberían establecer entre ladrillos para completar una integración medianamente comprensible. Solamente figuran algunas que indican por donde se van estableciendo aquellas interrelaciones, concordancias y discordancias, a la vez. La Teoría de Sistemas es la que establecería y resolvería esas relaciones entre los aspectos -ladrillos- diversos que presentan los distintos archivos aquí tomados como ejemplo. En general, siempre que se establezcan interrelaciones mutuas, sean éstas del tipo que sean, se habrá de aplicar la Teoría de Sistemas, que, asimismo, ha ampliado su ámbito de acción. Ahora se habla de Ciencia Sistémica y de Pensamiento Sistémico.

Obsérvese que se está utilizando la forma verbal condicional, en un intento de pensar que quizá si llegasen a aplicarse estas prácticas se podría alcanzar una integración vertical de ciertos archivos.

Sin embargo, una unificación -funcional- de los archivos españoles más conocidos se antoja un tanto imposible por las características peculiares de cada uno de ellos.

\section{RELACIÓN ENTRE EL INFORMACIONISMO Y LA INTEGRACIÓN VERTICAL DE ARCHIVOS}

Algo de lo que hasta ahora apenas se ha mencionado es la información que proporcionan los archivos. Conservar, ordenar, cuidar una colección de documentos conlleva un fin histórico en cuanto a la evolución del ser humano sobre nuestro planeta Tierra.

Dependiendo del tipo de documentos de que se trate, se podrá estudiar esa evolución en un aspecto u otro... Pero, indiscutiblemente, aún para realizar esos estudios de evolución histórica, habrá que sacar la información en aquellos documentos retenida.

La finalidad, primordial, de un archivo es facilitar información... Se vuelve al tema de la información como elemento imprescindible en nuestras vidas. En este caso, la información no es solamente nexo de unión entre los distintos tipos de documentos, sino también entre los conocimientos en ellos contenidos y los avatares existenciales de 
los propios individuos, deducibles de aquello. La información vuelve a ser hilo conductor entre los elementos particulares que componen la unidad de la ciencia Archivística, consolidando esa unificación al mismo tiempo que marcando su diferenciación interna.

La información vuelve a ser imprescindible en la relación del mundo interno de los archivos y el mundo exterior que los rodea y circunda. La información vuelve a ser... el paradigma principal que conduce a la formulación de una nueva teoría epistemológica llamada Informacionismo.

El Informacionismo encierra, en sí mismo, todo aquello relacionado con la información. Y esto es así, puesto que se ha considerado la información como su paradigma primero.

Los archivos, y su integración vertical, que son portadores y suministradores de información, se verán influenciados por el Informacionismo, de tal manera que llegarán a formar parte de sus postulados y principios.

La integración vertical de archivos conforma una parcela importante, y quizás imprescindible, en el desarrollo de las teorías y los enunciados de los supuestos epistemológicos constituyentes del propio Informacionismo.

Así pues, sería posible formular un enunciado que advirtiese: ¡Sin la integración vertical de archivos no se puede formular el Informacionismo!

\section{OBRAS DE REFERENCIA}

ARNZT, Helmut: Information und Hominisation: Grenzlinie der Entwicklung. Vorstudie zu einer Paläologie der Information. The Hague: FID 627, 1983.

BERGSTROM, R.M: "Man as an Information processor". Proceedings $44^{\text {th }}$ FID Conference and Congress. P. Hämäläinen; S. KOSKKIALA; A. J. Repo (eds.). Helsinki, 1988, 7-9.

BROTHMAN, Brien: "Ordenes de valores: cuestionando los términos teóricos de la práctica archivística". Tabula, 2007, 10: 27-58.

BUCKLAND, Michael: "Information as a thing". Journal of American Society of Information Science, 1991, 42, 5: 351-360.

CAPURRO, Rafael: Hermeneutik der Fachinformation. Freiburg; Munchen, 1986.

CURRÁS, Emilia: "Informationism and neuronal information assimilation", Information - Wissenschaft und Praxis, 2006, 4 (junio): 203-210; v.o.: "El metabolismo neuronal de la información". Conferencia pronunciada en la Real Academia de Farmacia y en el Ateneo de Madrid (1989).

CURRÁS, Emilia: "Vertical integration of Sciences: An approach to a different view of Knowledge Organization". Journal of Information Science, 2002, 28, 5: 417-426.

CURRÁS, Emilia: "Dialéctica en la organización del conocimiento". Organ. Conoc. Sist. Inf. Doc., 1999, 3: 23-43.

CURRÁS, Emilia: "An approach to application of Systematics to Knowledge Organization, finding new values and uses of Information". Proceedings $47^{\text {th }}$ FID Conference and Congress: Omiya, Saitama (Japan), $1994 .$. 
CURRÁS, Emilia: "Un nuevo concepto de información en la integración científica". 45th Conference and Congress of FID. La Habana: FID, sept. 1990, 19-38.

CURRÁS, Emilia: "Ciertos principios científico-filosóficos de las Ciencias de la Documentación. Rev. Univ. Complutense, 1983, 1, 4: 83-88.

CURRÁS, Emilia: “¿Estaremos en la época del Informacionismo”. Rev. Univ. Complutense, 1981, 2: 186-188.

DUFF, Wendy M.: "Normas de descripción archivística". Tabula, 2009, 11: 51-64.

EDO HERNÁNDEZ, Valentín: "Las clasificaciones de materias en Economía: valoración crítica y propuesta de una alternativa básica general. Revista Española de Documentación Científica, 2010, 33, 4: 600-623.

FARRADANE, Jason: "Knowledge, Information and Information Science". Journal of Information Science, 1980, 2: 75-80.

García Alsina, Montserrat: "Metodología para la implantación de la gestión del conocimiento a partir de técnicas documentales". Scire. Representación y Organización del Conocimiento, 2008, 14, 1: 43-61.

GARCÍA GONZÁLEZ, María; Más Bleda, Amalia: "Acceso a los fondos del Archivo General de Indias a través de la plataforma PARES (Portal de Archivos Españoles)". Naveg@merica,2010,5; web.

HENRICHS, Norbert: "Informationswissenschaft als angewandte Antropologie: Der Düsseldorfer Ansetz". Bucher für die Wissenschaft. Festschrift für Günter Gattermann. Munchen, etc: K.G. Saur, 1994, 445-461.

MARTÍN POZUELO, M. Paz: "Prospectiva archivística: nuevas cuestiones, enfoques y métodos de investigación científica". Revista Española de Documentación Cientifica, 2010, 33, 2; web.

MARTÍNEZ GARCÍA, Luis: "La génesis de los archivos nacionales españoles". Boletín de la ANABAD, 2006, 56, 2: 49-101.

MIKHAILOV, Alexander I.: "Science as a System of ciclic process of generation, processing, accumulation and transfer of Scientific Information". Theoretical Problems of Informatics: Place of Information in the Global Problems of the World. Moscow: VINITI, FID 659, 1982.

SILVEIRA ZARAGOÇA, F.J.: "Consideraçoes gerais sobre a problematica en Informaçao". Revista Española de Documentación Científica, 1980, 3, 2: 159-168. 